\title{
Solutions in Search of Problems
}

\author{
Jeremy F. Alm and David A. Andrews
}

A course in linear algebra is a central part of almost every undergraduate curriculum in mathematics. It is an important course for graduateschool-bound majors, as well as for science and engineering majors. For many students, it is a first introduction to formal proof.

Yet many professors and students are partially or wholly dissatisfied with undergraduate linear algebra courses. The two authors didn't like the courses we took as students, and the first author has been mostly disappointed with the two that he has taught. Our chief complaint-and there are many others who share this sentiment-is that the course doesn't "go anywhere" but is built around a loose assemblage of techniques. One starts with systems of linear equations, and fifteen weeks later finishes (often) with diagonalization of matrices. Then the course ends. This path is set out mostly by consensus: the undergraduate linear algebra course is said to be "packed", meaning that there is a lot of material that must be "covered", so we'd better get right on covering.

Timothy Gowers's essay "The two cultures of mathematics" [2] distinguishes between two types

Jeremy F. Alm is assistant professor of mathematics at Illinois College. His email address is $\mathrm{a} 7 \mathrm{~m}$. academic@gmai1.com.

David A. Andrews is assistant professor of mathematics at the University of Dallas. His email address is andrews@uda11as.edu.

Members of the Editorial Board for Doceamus are: David Bressoud, Roger Howe, Karen King, William McCallum, and Mark Saul.

DOI: http://dx.doi.org/10.1090/noti870 of mathematicians, the theory builders and the problem solvers:

If you are unsure to which class you belong, then consider the following two statements.

(i) The point of solving problems is to understand mathematics better.

(ii) The point of understanding mathematics better is to become better able to solve problems.

The significance of the "two cultures" phenomenon in the present context is this: most mathematics textbooks intended for undergraduate courses seem to have been written by theory builders for theory builders. They are organized linearly, so that an understanding of the $(n+1)$ st chapter, notation and all, depends on understanding the previous $n$ chapters. The techniques and tools are developed before any discussion of the problems one can solve with them (the so-called "applications"). The stated goal of the text has little meaning to the student and offers to them only much-delayed gratification. ${ }^{1}$ The outline of a course provided by such a text may seem sensible to a trained

\footnotetext{
${ }^{1}$ Sheldon Axler, in Linear Algebra Done Right [1], expresses the goal of "understanding the structure of linear operators." This goal is fascinating and worthwhile but will not mean much to the student at the beginning of a linear algebra course. Larry Smith, in the third edition of Linear Algebra [3], is quite explicit in stating his theory-building orientation: "The principal axis theorem [is] the first of two goals, and... a more or less direct path is followed to its proof." This path takes one through 329 pages.
} 
mathematician who sees how the ideas fit into "a tapestry in which all the concepts and skills are logically interwoven to form a single piece" [4]; however, this sensibility seems to be lost on many (or most) students, particularly those of a problem-solving bent.

As an alternative, we would suggest organizing a course or text around a set of problems. Finding a solution to each problem would be the goal for the students, around which the necessary concepts and mathematical machinery would be built. To be effective, each problem set would need to satisfy the following:

- The statement of each problem, including what counts as an answer to the problem, should be comprehensible to the student, perhaps after some initial setup involving concrete examples.

- The problems should seem uncontrived to the students so as not to be mistaken for make-work.

- The problems should be compelling to the instructor. Nothing helps student engagement more than an enthusiastic instructor.

- At least some of the problems should be "big", involving multiple ideas and techniques, so that the students have an opportunity to see the relationship between the diverse ideas. Some problems might take a few weeks to work to a solution.

- The problems should be small in number but chosen so that each major topic of a typical linear algebra course appears in at least one of the problems' solutions.

Consider, for instance, the following list of problems, which is not intended to be exhaustive but serves to illustrate that a large number of topics in a typical linear algebra syllabus can fall under the umbrella of just a few problems.

(i) Given $n$ linear equations in $n$ unknowns, how can you tell

(a) whether a solution exists, and

(b) if that solution is unique?

(ii) Given a list of students and their preferred courses, how can a course schedule be created to minimize conflicts?

(iii) Given a transition matrix that represents [insert your favorite discrete Markov process here], what is the long-term behavior of a state vector under continued application of the transition matrix?

(iv) Given a "tilted" ellipse, such as $x^{2}+2 x y+$ $2 y^{2}=1$, how can you find orthogonal axes $u, v$ so that the ellipse is "un-tilted" on these axes?

(v) Given $k$ points in the plane, with no two vertically aligned, how can you construct a polynomial that passes through all the points? (vi) How can you find a polynomial (such as is stored on your calculator) that gives a good uniform approximation to the sine function on $\left[\frac{-\pi}{2}, \frac{\pi}{2}\right]$ ?

Finally, although we are focusing explicitly on linear algebra in this article and in our problem list above, similar advice clearly applies, mutatis mutandis, to other disciplines. We imagine, for example, a "textbook" for statistics (the second author's discipline) that presents carefully chosen datasets and questions through which the techniques of statistics are introduced. It is our experience that many statistics books at the elementary level take an approach close to this, but the traditional "mathematical statistics" texts take a theory-building approach.

We certainly want students to understand mathematics better, and as Gowers notes, having them solve problems is a good means to this end. Students might benefit even more if we help them to see that good problems - and the discovery of their solutions!-can in themselves be worthwhile ends. Organizing a linear algebra course around significant problems would provide students with concrete and meaningful goals: more concrete than "understanding linear algebra" and more meaningful than "passing the next exam". It would also provide a more rewarding course for students of a problem-solving inclination, against whom the deck is currently stacked.

\section{References}

1. SHELDON AXLER, Linear Algebra Done Right, Undergraduate Texts in Mathematics, Springer-Verlag, New York, 1996.

2. W. T. Gowers, The two cultures of mathematics. In Mathematics: Frontiers and Perspectives, Amer. Math. Soc., Providence, RI, 2000, pp. 65-78. Available online at http: //www. dpmms . cam.ac.uk wtg10/2cu1tures. ps.

3. LARRY SMITH, Linear Algebra, Undergraduate Texts in Mathematics, Springer-Verlag, New York, third edition, 1998.

4. H. WU, The mis-education of mathematics teachers, Notices Amer. Math. Soc. 58 (2011), no. 3, 372-384. 